\title{
Erratum to: Le cancer du sein métastatique après la première ligne de chimiothérapie \\ Erratum to: Oncologie DOI:10.1007/s10269-011-2085-x
}

Les auteurs de l'article : Le cancer du sein métastatique après la première ligne de chimiothérapie souhaitent apporter des modifications à leur article, en page 656, volume 13, numéro 10-11, novembre 2011.

Il fallait lire :

[...] chez $96 \%$ des patientes [5]. Cette étude qui a inclus 762 patientes est positive en survie globale : 13,1 versus $\mathbf{1 0 , 6}$ mois, $p=\mathbf{0 , 0 4 1}, \mathrm{HR}=\mathbf{0 , 8 1}$. Ce traitement est donc un standard en troisième ligne ou plus (niveau IA).

L'étude Embrace est une étude de supériorité.

L'AMM concerne également le cancer du sein localement avancé. 